\title{
Approximation of the actual spatial distribution of the b-matrix in diffusion tensor imaging with bivariate polynomials
}

\author{
Krzysztof Kłodowski \\ Faculty of Physics and Applied Computer Science \\ AGH University of Science and Technology \\ in Kraków \\ al. Mickiewicza 30, 30-059 Kraków, Poland \\ Email: klodowski@fis.agh.edu.pl
}

\author{
Piotr Łukasik, Artur T. Krzyżak \\ Faculty of Geology, Geophysics and Environmental Protection \\ AGH University of Science and Technology \\ in Kraków \\ al. Mickiewicza 30, 30-059 Kraków, Poland
}

\begin{abstract}
The aim of this work was to find an analytical expression describing the b-matrix spatial distribution (BSD) in diffusion tensor imaging, obtained by means of simple calibration to a water isotropic phantom.

The bivariate second degree polynomial function was fitted for the complete set of spatially distributed b-matrix elements derived through measurements on a 3 Tesla clinical scanner.

Smooth, noise free b-matrices were obtained with clear patterns of systematic errors. Diffusion tensor eigenvalues were derived with much better accuracy than for previous BSD calibration. The proposed approach does not require many averages during the acquisition of the phantom and thus can shorten the BSD calibration.
\end{abstract}

\section{INTRODUCTION}

D IFFUSION Magnetic Resonance Imaging (dMRI) which appeared in the eighties and quickly developed towards Diffusion Tensor Imaging (DTI) [1] [2], has found number of clinical applications. It became very successful tool for neurological structural and functional imaging [3]. However, DTI is intrinsically prone to the numerous artifacts [4], which makes any quantitative comparison of the images obtained by different scanners questionable.

In order to calculate a diffusion tensor one has to acquire series of images, each with a different diffusion sensitizing gradient applied. The gradients can differ in both magnitude and orientation. Information about diffusion gradient schemes is stored in a b-matrix. The most common way of deriving the b-matrix is an approximated analytical calculation of each element. However, the applied diffusion gradients interact with other magnetic field gradients applied during the imaging sequence and thus the resultant gradients differ from what was expected. Variations of the magnetic susceptibility across the sample volume can also affect the b-matrix [5].

Several methods to partially solve such problems were proposed. Some of them introduced bipolar gradients which cancel out the cross-terms between the applied gradients [6], [7]. The other focused on choosing the best gradient encoding

Research financed by the National Centre of Research and Development (contract No. PBS2/A2/16/2013) scheme [8], [9], [10]. There were also post processing methods [11] and phantom calibration techniques [12] suggested.

A recent report revealed that for particular imaging sequence parameters the errors superimposed on the b-matrix have a systematic character and can be reduced through a calibration procedure [13]. The solution is based on the derivation of the b-matrix spatial distribution (BSD) which substitutes the standard b-matrix, constant for an entire volume. The BSD method improves the accuracy of diffusion measurements, but since the calibration is based on a real data, the derived distribution of the b-matrix is always biased with noise.

In this paper we present a procedure of deriving the noise free approximation of the actual spatial distribution of the bmatrices. The results are compared with both standard DTI and hitherto BSD-DTI.

\section{MATERIALS AND METHODS}

Standard diffusion tensor imaging of a water isotropic phantom was performed on a 3 Tesla clinical scanner. Six diffusion gradient directions were applied and the b-value was set to $1000 \mathrm{~s} / \mathrm{mm}^{2}$. The imaging was done in axial orientation, 25 interleaved slices with voxel size $1 \times 1 \times 3 \mathrm{~mm}$ were taken, with number of averages set to 4 . The acquisition was repeated then for the BSD calibration purposes.

The spatial distribution of the b-matrices was obtained through simple calibration to water phantom. The experiments were carried out in stable thermal conditions in temperature of $21^{\circ} \mathrm{C}$. The diffusion tensor eigenvalues for an isotropic water phantom in constant temperature are all equal and can be derived theoretically from the Einstein-Smoluchowski equation [14]:

$$
\left\langle r^{2}\right\rangle=6 D t
$$

where: $\left\langle r^{2}\right\rangle$ - is a mean square displacement, $D-$ is a diffusion coefficient, and $t-$ is a diffusion time. The off-diagonal elements of the tensor can be assumed equal to zero, since they describe the rotations of the diffusion ellipsoid being in this case spherically symmetrical. With the above assumptions 


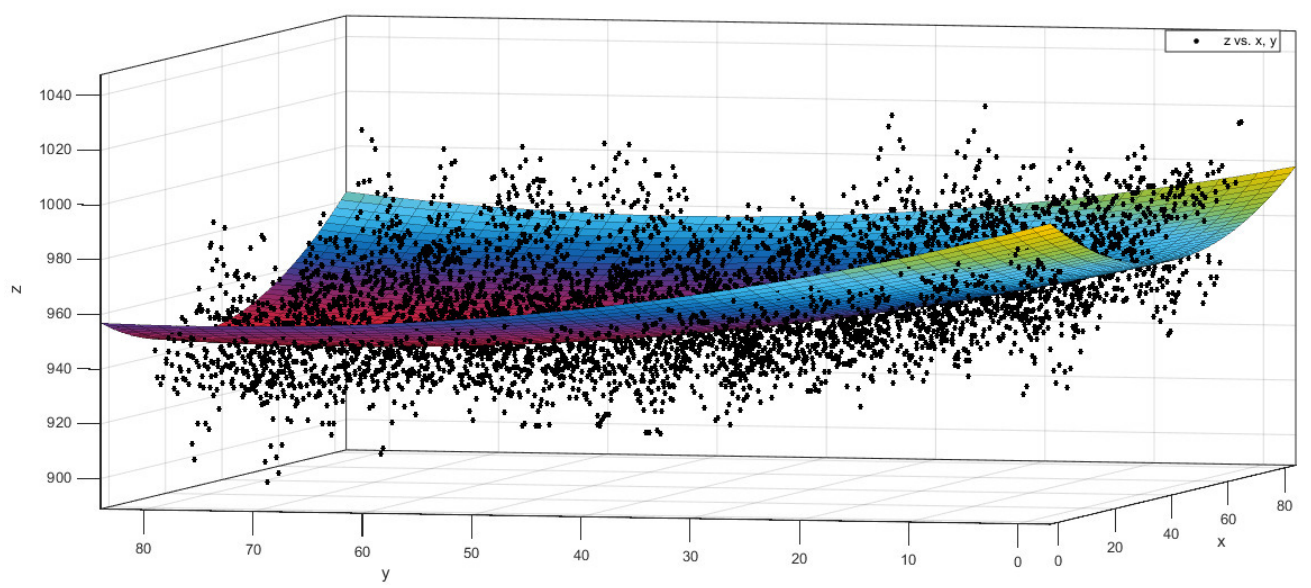

Fig. 1. Plot of bivariate polynomial fitted to data. In this case the diffusion gradient was applied solely along the $\mathrm{x}$ axis. The expression of the fitted function is the following: $f(x, y)=992.5-1.021 x-1.043 y+0.013 x^{2}+0.004 x y+0.007 y^{2}$.

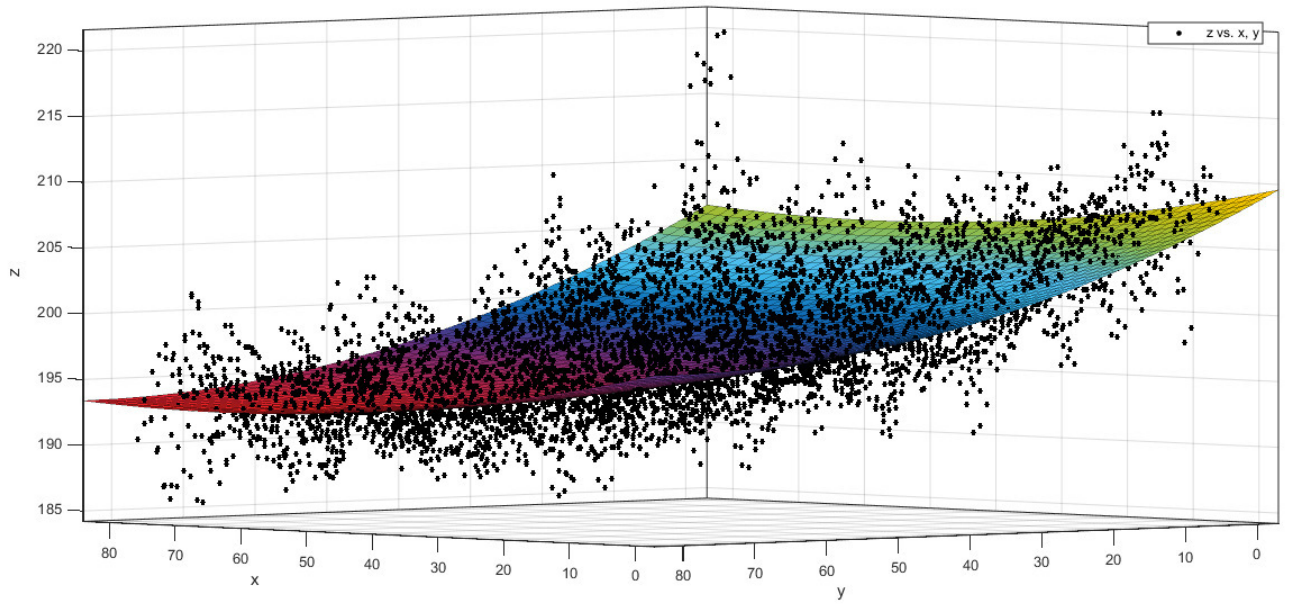

Fig. 2. Plot of bivariate polynomial fitted to data. In this case the dominating diffusion gradient was applied along the $\mathrm{y}$ axis with a nonzero $\mathrm{x}$ component. The expression of the fitted function is the following: $f(x, y)=208.4-0.1145 x-0.2833 y+0.0009 x^{2}-0.0001 x y+0.0017 y^{2}$.

the spatial distribution of the b-matrix elements for each voxel can be derived from Stejskal-Tanner equation [15].

$$
\ln \left(\frac{S_{x}}{S_{0}}\right)=-\mathbf{b}: \mathbf{D}
$$

where: $S_{x}$ - is a signal intensity in particular voxel measured with $x$ diffusion gradient applied, $S_{0}$ - is a signal intensity in particular voxel measured without diffusion gradient applied, b - is a b-matrix in particular voxel, $\mathbf{D}$ - is a diffusion tensor in particular voxel. The colon indicates a generalized dot product defined as:

$$
\mathbf{b}: \mathbf{D}=\sum_{i, j} b_{i j} D_{i j}
$$

where $b_{i j}$ and $D_{i j}$ are particular b-matrix and diffusion tensor elements, respectively.
The complete set of b-matrices derived from the above formulas consisted of 900 b-maps (25 slices times 6 b-matrix elements times 6 diffusion gradients).

An 80 × 80 pixel square region of interest (ROI) R1 inscribed in the circle of the phantom's axial projection was chosen for the analysis.

Theoretically each element of the b-matrix should be a scalar value derived from the sum of various quadratic gradient terms. The number of terms depends on what is really taken into account in calculating the b-matrix, but the diffusion gradients are always dominating, because of their relatively big strength. The influence of the imaging gradients is of secondary importance. Cross-terms between various types of gradients may also appear. Due to imperfections of the gradients, their nonlinearity and interactions between them the actual b-matrix should be described by a superposition of quadratic functions instead of a single constant value.

The actual character of the b-matrix may be expected to be 
TABLE I

COMPARISON OF THE MEAN EIGENVALUES AND THEIR STANDARD DEVIATIONS OBTAINED BY MEANS OF THE THREE APPROACHES. THE IMPROVEMENT FACTOR $\mathrm{K}_{\mathrm{SD}}$ IS EQUAL TO THE RATIO OF STANDARD DEVIATION OF STANDARD DTI TO THE SD OF A PARTICULAR APPROACH.

\begin{tabular}{llll}
\hline Experiment & $\mathrm{D}\left[\mathrm{mm}^{2} / \mathrm{s}\right]$ & $\mathrm{sd}\left[\mathrm{mm}^{2} / \mathrm{s}\right]$ & $\mathrm{K}_{\text {sd }}$ \\
\hline DTI & $1.9822 \times 10^{-3}$ & $5.73 \times 10^{-5}$ & 1.00 \\
BSD & $1.9984 \times 10^{-3}$ & $5.42 \times 10^{-5}$ & 1.05 \\
A-BSD & $1.9973 \times 10^{-3}$ & $3.92 \times 10^{-5}$ & 1.46 \\
\hline
\end{tabular}

described by a superposition of quadratic functions instead of a single constant value.

In order to fit a second order bivariate polynomial function:

$$
f(x, y)=a_{0}+a_{1} x+a_{2} y+a_{3} x^{2}+a_{4} x y+a_{5} y^{2},
$$

to all the 900 ROIs, a python script using least-squares fitting procedure was written.

The initial b-matrix values within the ROIs were substituted with values derived from the fitted functions. Eventually, diffusion tensors were calculated in three ways:

1) Standard DTI - using single b-matrix, constant in the entire ROI.

2) BSD - incorporating spatial distribution of the b-matrix, derived directly from the calibration procedure.

3) Actual BSD (A-BSD) - with usage of b-matrix spatial distribution derived from the fitted polynomial. The tensors were calculated for a circle ROI R2 inscribed in the R1 used for the fitting.

\section{RESULTS}

The calculated diffusion tensors were diagonalized with LU decomposition method in order to obtain the eigenvalues. For each of the 25 slices mean eigenvalue in R2 and its standard deviation were calculated. The results for selected slices together with the improvement factors $\mathrm{K}_{\mathrm{sd}}$ are presented in table I. Mean eigenvalues are quite similar for all the three approaches, however, standard deviations differ. The lowest value of sd, which indicates the best accuracy, was obtained for the A-BSD approach using the fitting procedure.

Plots of exemplary bivariate polynomial fits obtained for two different gradient directions are depicted in figures 1 and 2, respectively. The first corresponds to the diffusion gradient applied solely in $\mathrm{x}$ direction, whereas the latter, to the stronger component in $y$ direction and weaker, nonzero component in $x$.

Two dimensional maps of the fitted b-matrices for selected slices are depicted in figures 3 and 4 .

\section{DISCUSSION AND CONCLUSIONS}

The described procedure resulted in a noticeable improvement of the diffusion tensor eigenvalues homogeneity for the isotropic water phantom. The analysis was done in axial direction in which distortions in b-matrices are the smallest, accordingly to the previous studies [13] [16]. Thus also the $K_{\mathrm{sd}}$ improvement factor is relatively small. However, fitting the bmatrix data to the bivariate polynomial function decreases the

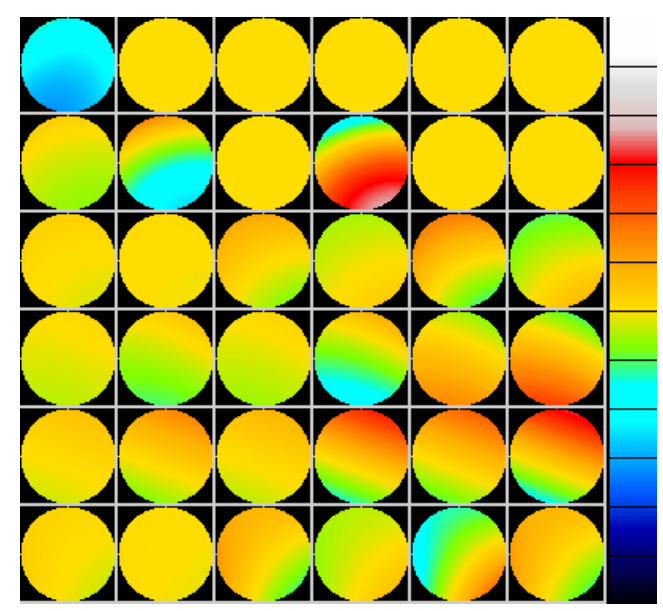

Fig. 3. Two dimensional map of b-matrix elements corrected with fitted bivariate polynomials. Each row represents one of the diffusion gradient directions, and each column is a b-matrix element in order: $b_{\mathrm{xx}}, \mathrm{b}_{\mathrm{yy}}, \mathrm{b}_{\mathrm{zz}}$, $b_{x y}, b_{x z}, b_{y z}$. The maps correspond to the middle slice, positioned in the isocenter of the scanner. The scale ranges from $-50 \mathrm{~s} / \mathrm{mm}^{2}$ to $50 \mathrm{~s} / \mathrm{mm}^{2}$. The map is differential, i.e. from each element standard, constant $b_{i j}$-value was subtracted.

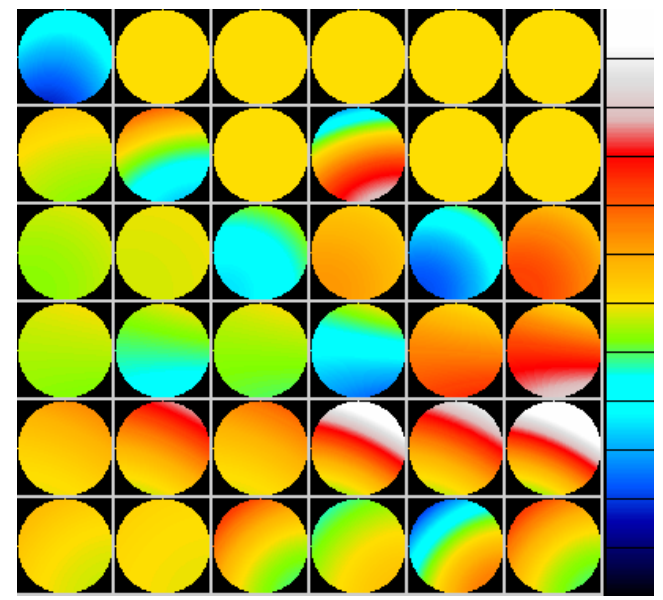

Fig. 4. Two dimensional map of b-matrix elements corrected with fitted bivariate polynomials. Each row represents one of the diffusion gradient directions, and each column is a b-matrix element in order: $b_{x x}, b_{y y}, b_{z z}$, $b_{x y}, b_{x z}, b_{y z}$. The maps correspond to the 25 slice, the farthest from the isocenter of the scanner. The scale ranges from $-50 \mathrm{~s} / \mathrm{mm}^{2}$ to $50 \mathrm{~s} / \mathrm{mm}^{2}$. The map is differential, i.e. from each element standard, constant $b_{i j}$-value was subtracted.

mean eigenvalue standard deviation to the approximately one third of the sd obtained through hitherto BSD calibration.

The analyzed dataset is characterized by a relatively high noise level, due to only 4 averages. Application of the fitting procedure visibly smooths the b-matrix spatial distribution and emphasizes the shape of the systematic errors. Figures and the equations of the fitted functions show that $\mathrm{a}_{3}$ coefficient is an order of magnitude bigger than $\mathrm{a}_{5}$ when the gradient direction was set along $\mathrm{x}$ axis and on the contrary the $\mathrm{a}_{5}$ coefficient is an order of magnitude bigger than $\mathrm{a}_{3}$ when the gradient is directed mostly along the y axis. The linear coefficients of the fits are also not negligible. It corresponds to the already 
discussed in this paper theoretical underpinning of the bmatrix. Complicated, by number of terms which are difficult to take into account in the analytical derivations (including crossterms), the structure of the b-matrix in the presented approach is revealed in its final shape through an experiment.

The two dimensional maps of the b-matrix elements depict the shape and intensity of the systematic errors. Comparison of figures 3 and 4 confirms the best homogeneity of the bmatrices in the isocenter of the scanner (most of the terms in figure 3 are close to yellow color corresponding to the assumed standard value of $b_{i j}$ element), and intensification of the distortions while moving towards the edges of the scanner (fig 4.).

In conclusion the presented method improves the BSD approach to DTI further. It enables one to derive noise-free spatial maps of b-matrices even from a dataset with relatively high noise level. This gives hope for shortening the BSD calibration time. Second degree bivariate polynomials turn out to define well the b-matrix elements according to the theory.

The presented approach successfully excludes the systematic errors affecting the accuracy of diffusion tensor derivation and opens the path for truly quantitative diffusion tensor measurements independent of a chosen scanner and imaging sequence.

\section{ACKNOWLEDGMENTS}

The work was financed by the National Centre of Research and Development, contract. No. PBS2/A2/16/2013 and contract No. STRATEGMED2/265761/10/NCBR/2015.

K. K. would like to thank the Marian Smoluchowski Cracow Scientific Consortium - KNOW for their support.

\section{REFERENCES}

[1] P. J. Basser, J. Mattiello, and D. LeBihan, "MR diffusion tensor spectroscopy and imaging." Biophysical Journal, 1994. [Online]. Available: http://www.ncbi.nlm.nih.gov/pmc/articles/PMC1275686/

[2] - "Estimation of the effective self-diffusion tensor from the NMR spin echo," Journal of Magnetic Resonance. Series B, 1994.

[3] D. Le Bihan and H. Johansen-Berg, "Diffusion MRI at 25: Exploring brain tissue structure and function," NeuroImage, 2012. doi: 10.1016/j.neuroimage.2011.11.006. [Online]. Available: http://www. sciencedirect.com/science/article/pii/S1053811911012857

[4] J.-D. Tournier, S. Mori, and A. Leemans, "Diffusion tensor imaging and beyond," Magnetic Resonance in Medicine, 2011. doi: 10.1002/mrm.22924. [Online]. Available: http://onlinelibrary.wiley. com.atoz.wbg2.bg.agh.edu.pl/doi/10.1002/mrm.22924/abstract
[5] P. J. Basser and D. K. Jones, "Diffusion-tensor MRI: theory, experimental design and data analysis - a technical review," NMR in Biomedicine, 2002. doi: 10.1002/nbm.783. [Online]. Available: http://onlinelibrary.wiley.com/doi/10.1002/nbm.783/abstract

[6] M. Neeman, J. P. Freyer, and L. O. Sillerud, "A simple method for obtaining cross-term-free images for diffusion anisotropy studies in NMR microimaging," Magnetic Resonance in Medicine, 1991. doi: 10.1002/mrm.1910210117. [Online]. Available: http://onlinelibrary.wiley.com.wiley-online-library.wbg2.bg. agh.edu.pl/doi/10.1002/mrm.1910210117/abstract

[7] A. L. Alexander, J. S. Tsuruda, and D. L. Parker, "Elimination of eddy current artifacts in diffusion-weighted echo-planar images: The use of bipolar gradients," Magnetic Resonance in Medicine, 1997. doi: 10.1002/mrm.1910380623. [Online]. Available: http://onlinelibrary.wiley.com.wiley-online-library.wbg2.bg. agh.edu.pl/doi/10.1002/mrm.1910380623/abstract

[8] K. M. Hasan, D. L. Parker, and A. L. Alexander, "Comparison of gradient encoding schemes for diffusion-tensor MRI," Journal of Magnetic Resonance Imaging, 2001. doi: 10.1002/jmri.1107. [Online]. Available: http://onlinelibrary.wiley.com.atoz.wbg2.bg.agh. edu.pl/doi/10.1002/jmri.1107/abstract

[9] D. K. Jones, "The effect of gradient sampling schemes on measures derived from diffusion tensor MRI: A Monte Carlo study," Magnetic Resonance in Medicine, 2004. doi: 10.1002/mrm.20033. [Online]. Available: http://onlinelibrary.wiley.com.atoz.wbg2.bg.agh. edu.pl/doi/10.1002/mrm.20033/abstract

[10] A. Özcan, "(Mathematical) Necessary conditions for the selection of gradient vectors in DTI," Journal of Magnetic Resonance, 2005. doi: 10.1016/j.jmr.2004.10.013. [Online]. Available: http://www. sciencedirect.com/science/article/pii/S1090780704003544

[11] M. E. Bastin, "Correction of eddy current-induced artefacts in diffusion tensor imaging using iterative cross-correlation," Magnetic Resonance Imaging, 1999. doi: 10.1016/S0730-725X(99)00026-0. [Online]. Available: http://www.sciencedirect.com/science/article/pii/ S0730725X99000260

[12] M. A. Horsfield, "Mapping eddy current induced fields for the correction of diffusion-weighted echo planar images," Magnetic Resonance Imaging, 1999. doi: 10.1016/S0730-725X(99)00077-6. [Online]. Available: http://www.sciencedirect.com/science/article/pii/S0730725X99000776

[13] A. T. Krzyżak and Z. Olejniczak, "Improving the accuracy of PGSE DTI experiments using the spatial distribution of b matrix," Magnetic Resonance Imaging, 2015. doi: 10.1016/j.mri.2014.10.007. [Online]. Available: http://www.mrijournal.com/article/S0730725X1400318X/abstract

[14] A. Einstein and R. Fürth, Investigations on the theory of Brownian movement, New York, N.Y., 1956.

[15] E. O. Stejskal and J. E. Tanner, "Spin Diffusion Measurements: Spin Echoes in the Presence of a Time-Dependent Field Gradient," The Journal of Chemical Physics, 1965. doi: 10.1063/1.1695690. [Online]. Available: http://scitation.aip.org/content/aip/journal/jcp/42/1/10.1063/1. 1695690

[16] K. Kłodowski and A. T. Krzyżak, "Innovative anisotropic phantoms for calibration of diffusion tensor imaging sequences," Magnetic Resonance Imaging, 2016. doi: 10.1016/j.mri.2015.12.010. [Online]. Available: http://www.mrijournal.com/article/S0730725X15003057/abstract 